\title{
Memory for relational information across eye movements
}

\author{
LAURA A. CARLSON-RADVANSKY \\ University of Notre Dame, Notre Dame, Indiana
}

\begin{abstract}
Structural descriptions are hierarchical representations of a visual stimulus in terms of its parts and their relations. Previous research in which the retention of parts was examined has shown that structural descriptions can be used to represent information in transsaccadic memory. In three experiments, this idea was tested further by examining whether relational information is also maintained across saccades. Experiment 1, in which a same/different comparison task was used, showed that both part and relation information are retained across fixations in the same manner as within a fixation. Experiment 2 showed that such retention is impervious to instruction. Finally, Experiment 3, in which a new set of stimuli was used, demonstrated that both coordinate and categorical relations are maintained across saccades. The results indicate that structural descriptions can be used to represent information in transsaccadic memory in a manner similar to their use in visual short-term memory.
\end{abstract}

The visual world extends before us in all directions, but our eyes only pick up information from a limited portion of this expanse at any one time. To accommodate for this limitation, we make eye movements, in order to select particular objects or regions of interest. Periods of time in which the eyes are in motion are called saccades, and the intake of visual information during saccades is actively suppressed by the visual system (see, e.g., Matin, 1974). This restricts visual information input to periods of time in which the eyes are relatively still, called fixations. Despite this discontinuous input, we perceive the world as familiar (e.g., not filled with novel to-be-identified objects with each fixation) and stable (e.g., objects do not appear to move, even though their retinal positions change). How this perception of a stable visual world is achieved is a classical question in visual perception.

One approach to addressing this issue has been to examine the retention of information in transsaccadic memory (e.g., Irwin, 1991), a memory store that preserves information from one fixation for use during subsequent fixations. The general methodology used to examine transsaccadic memory employs the saccade-contingent display change technique, pioneered by McConkie and Rayner (1975), which isolates the presentation of a stimulus to a single fixation. For example, a figure is presented during a single eye fixation, and a saccade is elicited; upon the

This research was supported by a B/START grant from NIMH (NIH/1R03 MH56303-01) and by the Institute for Scholarship in the Liberal Arts, College of Arts and Letters, University of Notre Dame. I thank David Irwin for suggesting Experiment 3; John Henderson, Brad Gibson, G. A. Radvansky, Keith Rayner, Karl Verfaillie, and an anonymous reviewer for helpful comments; and James Brockmole and Kathleen Lattanzi for help in collecting the data. Correspondence concerning this article should be addressed to L. Carlson-Radvansky, Department of Psychology, University of Notre Dame, Notre Dame, IN 46556 (email: laura.c.radvansky.2@nd.edu). detection of the saccade onset, the first figure is erased, and a second figure is presented during the next eye fixation. The subject's task usually involves a comparative judgment about the two figures. This task requires transsaccadic memory, because the first figure is no longer present when the second figure appears (Irwin, 1992a).

Using such a paradigm, research has demonstrated that visual information is retained in transsaccadic memory (see Irwin, 1992b; Pollatsek \& Rayner, 1992, for reviews). For example, using a picture-naming paradigm, Pollatsek, Rayner, and Collins (1984) presented subjects with a preview line drawing in one fixation and a to-be-named target line drawing in a subsequent fixation. A significant naming time benefit was found when the preview was visually similar and conceptually dissimilar to the target (e.g., when the preview was a carrot and the target was a baseball bat). Similarly, Irwin (1991) showed that subjects could decide with good accuracy (about $70 \%$ ) whether a dot pattern presented in one fixation matched a second pattern presented in a subsequent fixation (see, also, Henderson, 1997; Irwin, Yantis, \& Jonides, 1983; O'Regan \& Levy-Schoen, 1983; and Rayner \& Pollatsek, 1983, for further supporting evidence against the retention of highly detailed point-by-point information in transsaccadic memory using a variety of stimuli).

Although the abstract nature of visual information has been rather firmly established, the level of representation at which visual information is retained remains uncertain. Irwin (1991) has shown that transsaccadic memory shares a number of properties with visual short-term memory, a memory store that exists within eye fixations (see, e.g., Phillips, 1974), and has suggested that they may in fact be the same memory structure. If so, the types of representations that are known to be used in visual short-term memory may also be used transsaccadically. Carlson-Radvansky and Irwin (1995) recently investigated this issue by asking whether structural descriptions 
(Gottschaldt, 1926/1967; Palmer, 1977; Sutherland, 1968 ) are used in transsaccadic memory in the same manner in which they are used in visual short-term memory. According to Palmer, a structural description is a hierarchical representation of a figure, in which nodes correspond to certain parts of the figure and connections between the nodes represent the relations among the parts. The representation is selective, in that only parts defined by organizational properties, such as connectedness and proximity, are explicitly encoded in the hierarchy. Accordingly, "good" parts are those that are directly represented as nodes in the structural description of the figure, and "bad" parts are those that are not directly represented, but whose presence can be verified only by constructing the part from elements dispersed across nodes.

To assess whether structural descriptions are used in transsaccadic memory, Carlson-Radvansky and Irwin (1995) employed a number of tasks using the saccadecontingent display technique, in which two figures were presented in separate fixations. The results showed significant benefit (faster and more accurate responses) for making decisions about stimuli containing "good" parts, as compared with "bad" parts, as designated by independent ratings of part goodness. This pattern suggested the use of structural descriptions (Palmer, 1977).

Given that structural descriptions encode not only parts of a figure but also the relations among the parts, if structural descriptions are used to represent abstract visual information in transsaccadic memory, relational information should also be retained from one fixation to the next. Moreover, given the similarities between transsaccadic memory and visual short-term memory (Carlson-Radvansky \& Irwin, 1995; Irwin, 1991), such retention should be similar both across fixations and within a fixation.

\section{EXPERIMENT 1}

Experiment 1 employed a transsaccadic same/different comparison task in which two figures were presented in different fixations, and the task was to decide whether they were the same or different. Of central interest is the relationship between the figures presented on each trial. Four conditions were constructed, using stimuli in which global structure and local parts were crossed, as in the global/local paradigm (see, e.g., Kimchi, 1988, 1992; Navon, 1977). The conditions are illustrated in Figure 1. In row 1 , the stimuli have the same parts and the same relations (henceforth, the all-same condition). In row 2, they have the same parts but different relations (henceforth, the same-part condition). In row 3 , they have different parts but the same relation (henceforth, the samerelation condition). Finally, in row 4 , they have different parts and different relations (henceforth, the no-same condition). The correct response for row 1 is same; the correct response for rows $2-4$ is different.
Such stimuli have been classified as Type P (Pomerantz, 1983), because the global/local relationship is based only on the position of the local elements, and not on their identity. These stimuli are also considered few-element figures, because they contain fewer than seven local parts; thus, these parts serve as figural elements, rather than as texture elements (Kimchi, 1988, 1992). For these stimuli, the relational information that specifies how the local parts are configured could be represented within a structural description of the figure at one of two locations. First, consistent with Palmer (1977), relational information may specify the relations among the local parts, thus appearing at the same level as the local parts in the hierarchy. For example, for the first stimulus shown in Figure 1 , the node at the top level of a structural description would correspond to the whole figure (i.e., the Greek letter gamma, $\Gamma$ ), and the next level down would correspond to the individual trapezoids, with the relations (e.g., on top of, to the right of) specified among the individual elements. Second, given the difficulty in segregating relations from parts (Kimchi, 1992), relational information could also be taken as corresponding to the global structure or configuration of the figure and, thus, be represented at the topmost level of the structural description. For example, for the first stimulus depicted in Figure 1, the top node might correspond to the global configuration of the Greek letter gamma $(\Gamma)$, the next level would consist of features that make up the global structure (i.e., horizontal line, vertical line, and right angle), and the next level would consist of the specific local elements that together constitute gamma (trapezoids). Importantly, regardless of whether it is considered as local relations or global structure, relational information is represented separately from the part information in a structural description, thus rendering these stimuli appropriate for an examination of the independent retention of parts and relations.

The critical comparisons for assessing retention of parts and relations involve the different trials. If relational information is maintained in transsaccadic memory, deciding that two stimuli are different when they share the same global relation but different local parts (row 3, same-relation condition) should be more difficult than deciding that they are different when they differ in both parts and relations (row 4, no-same condition). Similarly, if part information is maintained in transsaccadic memory (Carlson-Radvansky \& Irwin, 1995), deciding that two stimuli are different when they share the same parts but not the same relation (row 2, same-part condition) should be more difficult than deciding that they are different when they differ in both parts and relation (row 4, no-same condition). Such a pattern of faster response times as a function of increasing differences between stimuli has been well established, using a variety of stimuli (see Farell, 1985, and Nickerson, 1972, for reviews). 


\begin{tabular}{|c|c|c|c|c|c|}
\hline Pair Type & $\begin{array}{c}\text { First } \\
\text { Stimulus }\end{array}$ & $\begin{array}{l}\text { Second } \\
\text { Stimulus }\end{array}$ & $\begin{array}{c}\text { Experiment 1 } \\
\text { Responses }\end{array}$ & $\begin{array}{l}\text { Experiment } 2 \\
\text { Part- } \\
\text { Instruction } \\
\text { Responses }\end{array}$ & $\begin{array}{c}\text { Experiment 2 } \\
\text { Relation- } \\
\text { Instruction } \\
\text { Responses }\end{array}$ \\
\hline $\begin{array}{l}\text { All-Same: } \\
\text { Same parts } \\
\text { Same relation }\end{array}$ & & & Same & Same & Same \\
\hline $\begin{array}{l}\text { Part-Same: } \\
\text { Same parts } \\
\text { Different } \\
\text { relation }\end{array}$ & & & Different & Same & Different \\
\hline $\begin{array}{l}\frac{\text { Relation-Same }}{\text { Different Parts }} \\
\text { Same relation }\end{array}$ & & & Different & Different & Same \\
\hline $\begin{array}{l}\frac{\text { No-Same }}{\text { Different parts }} \\
\text { Different } \\
\text { relation }\end{array}$ & & & Different & Different & Different \\
\hline
\end{tabular}

Figure 1. Critical pair types for Experiment 1.

\section{Method}

\section{Subjects}

Eight subjects from the University of Notre Dame community were tested; they were compensated at a rate of $\$ 6$ for each hour of their participation.

\section{Stimuli}

The stimuli were in the spirit of those used in examining the processing of multidimensional stimuli (e.g., Garner, 1974; Santee \& Egeth, 1980) and in the processing of few-element figures within the global/local paradigm (e.g., Kimchi, 1988, 1992; Navon, 1977; Pomerantz, 1983). More specifically, there were four possible relations, constructed by connecting a subset of dots from an invisible $3 \times 3$ matrix. There were also four possible parts: triangles, trapezoids, diamonds, and pentagons. Each part subtended $1.5^{\circ}$ of visual angle at its widest or tallest point. The relations ranged from $2.9^{\circ}$ to $4.5^{\circ}$ in width and height. The parts and relations were crossed, producing 16 possible figures. Figure 2 shows the four relations, each with one set of parts.

\section{Apparatus}

The stimuli were presented on a Nec MultiSync 15-in. flat screen color monitor equipped with $\mathrm{P}-22$ phosphor. The vertical refresh rate was $72 \mathrm{~Hz}$, resulting in a delay of $0-14 \mathrm{msec}$ to erase the screen. Stimulus presentation and response collection were controlled by a Gateway 2000 Pentium computer. For eye movement monitoring, an Applied Science Laboratory Model 210 scleral reflectance eye tracker was used that sampled the left eye every millisecond.

The subjects made their responses by pressing hand-held switches. The subjects held the yes switch in their dominant hands and the no switch in their nondominant hands. Response time was measured from the onset of the second figure to the response. The sequence of trials was self-paced. The subjects used a bite-bar on the eye movement trials and a chinrest on the no eye movement trials, to help eliminate head movements.

\section{Design}

For each of the 16 figures there were 18 trials, broken down into 9 same trials with the same parts/same relation, and 9 different trials, 3 with same parts/different relation, 3 with different parts/same relation, and 3 with different parts/different relation. This yielded 288 trials. These were presented within each of four overlap conditions, two that involved eye movements, and thus invoked transsaccadic memory, and two that did not involve eye movements, and thus invoked visual short-term memory. These conditions enabled a comparison of the retention of relational information across these memory stores.

On the retinal overlap eye movement trials, the first figure was presented at central fixation, with a saccade target appearing $4.8^{\circ}$ to the left or the right. Upon elicitation of a saccade, the first figure was erased, and the second figure was presented at the saccade target's location, so that when the eyes landed, the second figure appeared at approximately the same retinal location as the first figure. On the spatial overlap eye movement trials, the first figure was presented within an invisible matrix centered at $4.8^{\circ}$ to the left or the right of a central fixation point. Upon elicitation of a saccade to the figure, the first figure was erased, and a second figure was presented at the same spatial location as the first, so that when the eyes landed, they would fixate the second figure. Note that the presentation of the second figure is foveal in both of these eye movement conditions, 

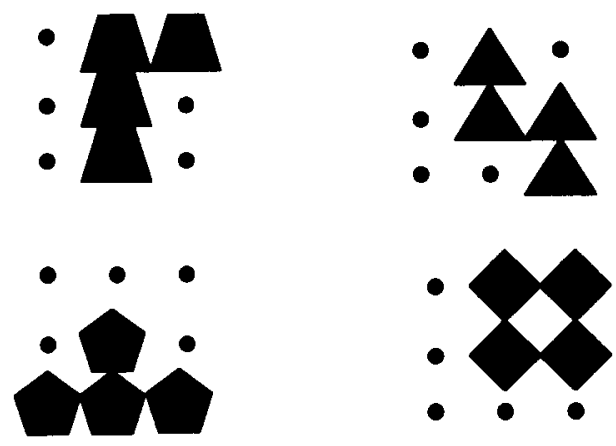

Figure 2. Sample stimuli showing the four possible local parts and the four possible global relations. These levels were crossed to produce the 16 possible stimuli used in Experiments 1 and 2.

whereas the presentation of the first figure is foveal in the retinal overlap condition and peripheral in the spatial overlap condition. Both types of trial were included because previous work within the global/local paradigm implicated eccentricity as a factor in determining the manner in which the global relations and local parts are processed (Lamb \& Robertson, 1988; Pomerantz, 1983). On the retinal + spatial overlap no eye movement trials, both figures were presented at central fixation, in the same retinal and spatial location. On the no overlap no eye movement trials, the first figure was presented to the peripheral retina and the second figure at central fixation (a different retinal and spatial location).

In all, the subjects performed 288 trials of each eye movement and no eye movement type, yielding a total of 1,152 trials. For the eye movement trials, these were randomly divided into four blocks of 72 trials each, with the subjects performing one block in each condition during an experimental session, with order counterbalanced. In an additional session, the subjects performed 288 trials of each type of the no eye movement trials. Note that eye movement sessions were always conducted before the no eye movement sessions, because the timing parameters of the saccades were needed to determine the stimulus duration for the first figure for the no eye movement trials.

\section{Procedure}

Each subject took part in an initial practice session and five experimental sessions. In all sessions, the subjects were told that they would be judging whether two figures were the same or different. The figures had to be exactly the same in order to be judged same. The subjects were told that their responses would be timed and that they should respond as quickly and as accurately as possible.

Practice session. The subjects performed one block of 72 no overlap no eye movement trials and 1-3 sets of 10 trials in each of the eye movement trials, to familiarize themselves with the sequence of events. The practice session used stimuli that did not appear in the experimental session.

Eye movement trials. In the first four experimental sessions, the subjects performed 72 trials of each eye movement trial type. The sequence of events constituting a retinal overlap trial is shown in Figure 3A and that constituting a spatial overlap trial in Figure $3 \mathrm{~B}$. Each eye movement trial began with a calibration routine in which a series of three fixation points $(+)$ appeared sequentially across the screen (see panel 1, Figures $3 \mathrm{~A}$ and $3 \mathrm{~B}$ ). The subject was instructed to fixate each point carefully. The first point appeared for $1,500 \mathrm{msec}, 8^{\circ}$ to the left of center $\left(-8^{\circ}\right)$, the second point at the center $\left(0^{\circ}\right)$, and the third point $8^{\circ}$ to the right of center $\left(+8^{\circ}\right)$. The average of the samples corresponding to time interval $900-1,000 \mathrm{msec}$ for each calibration point was calculated, and these means were used to calibrate the output of the eye tracker with spatial position.
After a successful calibration, the subject moved his or her eyes to a central fixation point that was presented for $1,500 \mathrm{msec}$ ( $\mathrm{sec}$ panel 2, Figures $3 \mathrm{~A}$ and $3 \mathrm{~B}$ ). The first figure appeared within an invisible matrix centered at $4.8^{\circ}$ to the left or the right periphery in the spatial overlap condition (see Figure 3B) or at fixation, with a plus sign serving as the saccade target in the left or the right periphery in the foveal retinal overlap condition (see Figure 3A). The subject was instructed to make an eye movement to the target in the periphery (either the figure or the plus sign) once the first stimulus had been identified (see panel 3, Figures $3 \mathrm{~A}$ and $3 \mathrm{~B}$, where the arrow represents the saccade). The detection of the saccade was made on the basis of a change in eye position in the same direction that exceeded $0.05 \% \mathrm{msec}$ for 3 consecutive msec. Upon saccade detection, the screen was blanked for $42 \mathrm{msec}$, followed by the presentation of the second figure at the target location (see panel 4, Figures $3 \mathrm{~A}$ and 3B). Response time was recorded from the detection of the saccade to the response and, thus, included the saccade duration.

In order for a trial to be considered acceptable, the following criteria had to be met. First, to ensure that the subjects were fixating on the initial figure, the starting fixation location had to be within $\pm 1.5^{\circ}$ of the central fixation point when the first figure appeared. Second, to ensure that the subjects had enough time to identify the first figure but not an exceedingly long time, the saccade latency had to be greater than $100 \mathrm{msec}$ and less than $1,000 \mathrm{msec}$. Third, the subject's eye movement had to land within the matrix in which the second figure was drawn. Fourth, saccade duration had to be greater than $22 \mathrm{msec}$ and less than $80 \mathrm{msec}$. The maximum duration was arbitrarily set to flag trials in which saccadic behavior was anomalous. The minimum duration was set to ensure that the subjects perceived the two figures in separate eye fixations and was based on the response lag of the eye tracker $(4-5 \mathrm{msec})$, the on-line calculation that a saccade had occurred $(3 \mathrm{msec})$, and the vertical retrace delay (at most, $14 \mathrm{msec}$ ). Following the recommendation of Irwin (1994; Irwin et al., 1983), a shutter test verified that the first figure did not persist beyond this 14-msec retrace delay. ${ }^{1}$ Fifth, response time had to be less than $5,000 \mathrm{msec}$. Together, these criteria ensured that the subjects were viewing the first and second figures in separate fixations and, thus, required the retention of information from the first figure in transsaccadic memory. In all, $80 \%$ of the trials were acceptable. Significantly more trials were acceptable in the spatial overlap condition ( $M=87 \%$; percent eliminated for criteria $1-5: 1 \%, 0 \%, 1 \%, 11 \%$, and $0 \%$, respectively) than in the retinal overlap condition ( $M=73 \%$; percent eliminated for criteria 1-5: $8 \%, 1 \%, 1 \%, 17 \%$, and $0 \%$, respectively). This was due mostly to the difficulty that the subjects had, in the retinal overlap condition, with moving off of the foveally presented figure; this resulted in small saccades that preceded the saccade to the target (criteria 1 and 4; see, also, Carlson-Radvansky \& Irwin, 1995).

No eye movement trials. In the last experimental session, the subjects performed 288 trials of each type of no eye movement trial. The sequence of events for a retinal + spatial overlap trial is shown in Figure 3C, and that for a no overlap trial in Figure 3D. The subjects were instructed to fixate the center fixation point throughout the trial. The first figure was presented $4.8^{\circ}$ to the left or the right periphery in the no overlap condition (see panel 1, Figure 3D) or at fixation for the retinal + spatial overlap condition (see panel 1, Figure 3C).

The timing parameters of the no eye movement trials were yoked for each subject to the timing of events in the eye movement trials, to ensure that the first stimuli were displayed for equivalent durations. Specifically, for the retinal + spatial overlap trials, the duration of the first stimulus was based on the mean saccade latency from the retinal overlap eye movement trials ( $M=320 \mathrm{msec}$ across subjects) because, on both of these types of trials, the first stimulus appeared foveally. For the no overlap trials, the duration of the first stimulus was based on the mean saccade latency from the spatial overlap trials ( $M=236 \mathrm{msec}$ across subjects) because, on both of these types of trials, the first stimulus appeared peripherally. Next, a blank 
A

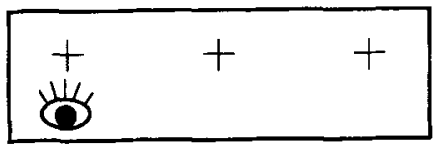

Calibration: Each point fixated for 1.5 seconds

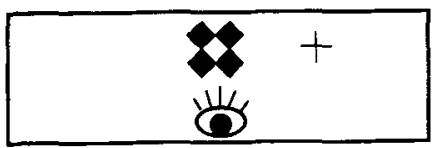

First figure appears at fixation with saccade target in periphery

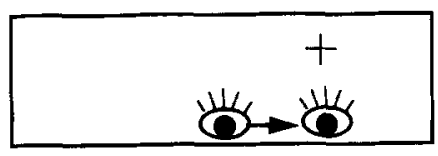

Upon saccade detection first figure disappears fol $42 \mathrm{~ms}$

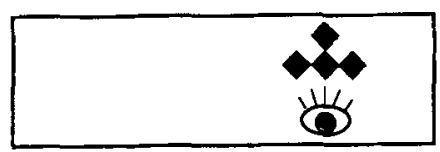

Second figure appears; response collected

C

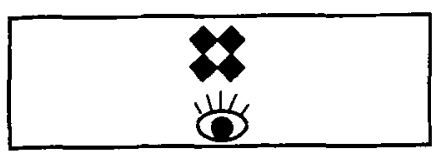

First figure appears at fixation for duration of mean saccade latency from retinal overlap trials

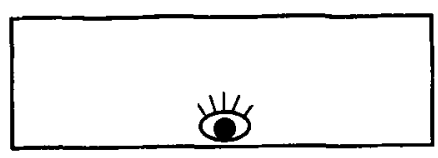

Screen is blank for $42 \mathrm{~ms}$

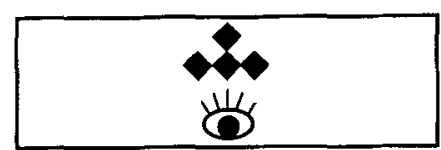

Second figure appears at fixation; response collected
B

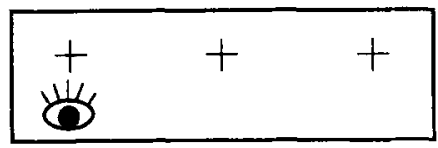

Calibration: Each point fixated for 1.5 seconds

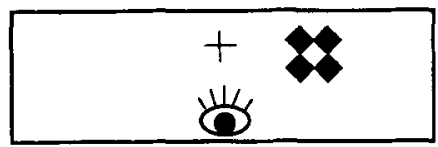

First figure appears in periphery with central fixation target

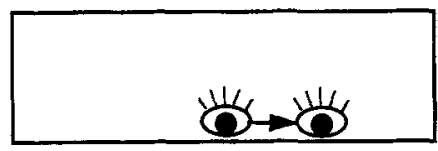

Upon saccade detection first figure disappears for $42 \mathrm{~ms}$

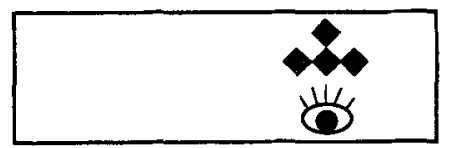

Second figure appears; response collected

D

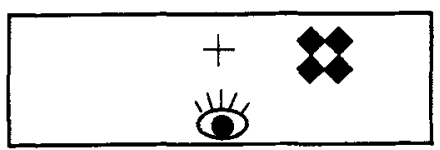

First figure appears in periphery for duration of mean saccade latency from spatial overlap trials

Screen is blank for $42 \mathrm{~ms}$
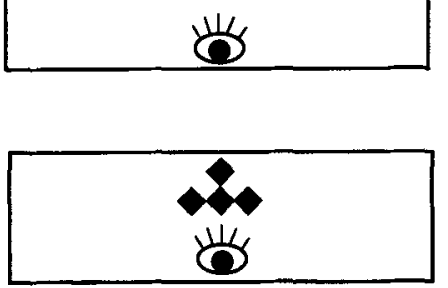

Second figure appears at fixation; response collected

Figure 3. Types of trials used in Experiments 1 and 2. Panel A illustrates the retinal overlap eye movement trials; panel B illustrates the spatial overlap eye movement trials; panel $C$ illustrates the retinal + spatial overlap no eye movement trials; and panel $D$ illustrates the no overlap no eye movement trials.

screen appeared for $42 \mathrm{msec}$, an interval meant to approximate the saccade duration in the eye movement trials (see panel 2, Figures $3 \mathrm{C}$ and $3 \mathrm{D}$ ). This was followed by the presentation of the second stimulus at the central fixation point (see panel 3 , Figures $3 \mathrm{C}$ and 3D). The subjects were instructed to decide as quickly but as accurately as possible whether the two stimuli were the same or different. Response time was measured from the presentation of the second stimulus to the response. Trials with response times greater than 5,000 msec were considered unacceptable (less than $1 \%$ of the data).

\section{Results and Discussion}

Table 1 presents the mean correct response times and percent correct, calculated as a function of stimulus pair type and overlap condition. The data of primary interest are the three pair types requiring a different response: the same-relation, same-part, and no-same conditions. If infor- mation about relations and parts are retained in transsaccadic memory, there should be significant interference, as revealed by longer response times and higher error rates for determining that two figures are different when they share either the same relations or the same parts, relative to when they share neither relations nor parts. Moreover, given the similarities between transsaccadic memory and visual short-term memory (CarlsonRadvansky \& Irwin, 1995; Irwin, 1991; Phillips, 1974), this pattern should be observed in both the eye movement conditions (an index of transsaccadic memory) and the no eye movement conditions (an index of visual shortterm memory) ${ }^{2}$

To test this idea, response times and percent correct were submitted to separate 3 (different pair types) $\times 4$ (overlap condition: retinal overlap eye movement, spa- 
Table 1

Mean Correct Response Times (in Milliseconds) and Percent Correct (PC) for Same and Different Responses Broken Down by Trial Type for Experiment 1

\begin{tabular}{|c|c|c|c|c|c|c|c|c|}
\hline \multirow[b]{4}{*}{ Trial Type } & \multicolumn{8}{|c|}{ Response and Stimulus Pair Type } \\
\hline & \multirow{2}{*}{\multicolumn{2}{|c|}{$\frac{\text { Same Response }}{\text { All-Same }}$}} & \multicolumn{6}{|c|}{ Different Responses } \\
\hline & & & \multicolumn{2}{|c|}{ Same-Part } & \multicolumn{2}{|c|}{ Same-Relation } & \multicolumn{2}{|c|}{ No-Same } \\
\hline & $M$ & $\mathrm{PC}$ & $M$ & $\mathrm{PC}$ & $M$ & $\mathrm{PC}$ & $M$ & $\mathrm{PC}$ \\
\hline \multicolumn{9}{|c|}{ Eye Movement Trials } \\
\hline Retinal overlap & 450 & 93 & 500 & 97 & 498 & 95 & 473 & 97 \\
\hline Spatial overlap & 411 & 96 & 472 & 97 & 472 & 94 & 448 & 98 \\
\hline \multicolumn{9}{|c|}{ No Eye Movement Trials } \\
\hline Retinal + spatial overlap & 418 & 98 & 499 & 97 & 515 & 97 & 488 & 97 \\
\hline No overlap & 438 & 98 & 531 & 96 & 535 & 94 & 510 & 98 \\
\hline
\end{tabular}

Note-All-same refers to a stimulus pair with the same parts and same relation; same-part refers to a stimulus pair with the same parts but different relation; same-relation refers to a stimulus pair with the same relation but different parts; no-same refers to a stimulus pair with different parts and different relations.

tial overlap eye movement, retinal + spatial overlap no eye movement, and no overlap no eye movement) repeated measures analyses of variance (ANOVAs). For these and all the subsequent analyses, a $p$ value of .05 was adopted for significance, unless otherwise indicated. Follow-up tests for significant main effects and interactions were based on critical differences required for significance that were calculated on the basis of $95 \%$ confidence intervals, using the error term for the main effect or interaction, as appropriate (Fisher, 1966; Loftus \& Masson, 1994).

\section{Different Response Times}

There was a significant effect of different pair type $\left[F(2,14)=34.3, M S_{\mathrm{e}}=169.1\right]$. On the basis of a critical difference of $14 \mathrm{msec}$ for significance, response times were significantly longer in the same-relation condition $(M=505 \mathrm{msec})$ than in the no-same condition $(M=$ $480 \mathrm{msec}$ ). This indicates interference in making the different response when the relation remained the same across the two stimuli, relative to when the relations differed across the two stimuli. This finding suggests that relational information was maintained. Response times were also significantly longer in the same-part condition $(M=$ $501 \mathrm{msec})$ than in the no-same condition $(M=480 \mathrm{msec})$. This indicates that part information was maintained, replicating Carlson-Radvansky and Irwin (1995), using different stimuli and a different task. There was no difference between the same-part condition $(M=501 \mathrm{msec})$ and the same-relation condition $(M=505 \mathrm{msec})$. Note that this only indicates that, for these stimuli, the part information and the relation information were equally salient; for other stimuli, relational or global information tends to be accessed prior to part or local information, as in the global precedence effect (see, e.g., Navon, 1977; see Kimchi, 1992, for a review).

There was no main effect of overlap condition $(F<2.2$, $p>.11)$, and more importantly, there was no significant interaction between pair type and overlap condition $(F<1)$, indicating similar pair type effects in both transsaccadic memory and visual short-term memory.

\section{Different Response Accuracy}

There was a significant effect of different pair type $\left[F(2,14)=4.3, M S_{\mathrm{e}}=10.8\right]$. On the basis of a critical difference of $4 \%$ for significance, accuracy did not significantly differ in pairwise comparisons across conditions; however, the pattern in the means was consistent with more difficulty in the same-relation $(M=95 \%)$ and same-part $(M=97 \%)$ conditions than in the no-same condition $(M=98 \%)$. There was no effect of overlap condition $(F<1)$, and although the interaction approached significance $\left(F=2.0, M S_{\mathrm{e}}=5.5, p=.09\right.$, with a critical difference of $2 \%$ for significance), any differences among overlap conditions were not contingent on the presence or absence of an eye movement (see Table 1).

\section{Same Responses}

For completeness, analyses conducted on same responses in the all-same condition, in which both parts and relations were maintained, revealed no effect of overlap condition in either the response times $(F=1.3, p<.3)$ or accuracy $(F=2.1, p<.14)$.

\section{Summary}

The response time and accuracy results indicate that both part and relational information are encoded within a fixation, maintained across transsaccadic and visual short-term memory, respectively, and interfere with the speed and accuracy with which different responses can be made. Such interference was of similar magnitude both within and across fixations.

\section{EXPERIMENT 2}

The goal of Experiment 2 was to determine the obligatory nature of the retention of parts and relations in transsaccadic memory. Experiment 1 demonstrated that parts 
and relations are encoded for the first stimulus and then invoked during the processing of the second stimulus. Indeed, the design of Experiment 1 was such that it required processing both features for determination of the correct response. In contrast, Experiment 2 examined the retention of parts and relations when the subjects were explicitly instructed to pay attention to one feature while ignoring the other. If the encoding and maintenance of these features is under strategic control, the subjects should be able to selectively attend to either the parts or the relations. However, if the encoding of the stimulus in terms of its parts and relations is obligatory and such information is represented in a structural description that is carried across a saccade, there should remain an influence of the irrelevant feature on responses to the relevant feature.

For example, some subjects were told that they would be shown two stimuli that consisted of parts in some relation (see the Relation-Instruction column in Figure 1). They were instructed to respond same when the parts remained constant across the two stimuli, regardless of whether the relations among the parts was the same or different. Selective encoding of the parts should result in no difference in the speed or accuracy of making same judgments in the all-same and the part-same conditions. That is, whether the relation among the parts remains the same (as in the all-same condition) or differs (as in the part-same condition) should not influence performance. However, if subjects automatically encode and maintain the relations, responses when the relations are constant across the stimuli should be faster and more accurate than responses when the relations change. Such facilitation from the congruence of the irrelevant dimension has been found on same responses in a number of studies using multidimensional stimuli (e.g., Besner \& Coltheart, 1975; Garner, 1988; Hawkins \& Shigley, 1972; Miller \& Bauer, 1981; Santee \& Egeth, 1980; for a review, see Farell, 1985). Similar reasoning can be applied to the obligatory nature of the retention of part information for subjects told to respond same when the relations remain the same across the two parts, regardless of whether the parts were the same (see the Part-Instruction column in Figure 1).

It is also possible to compare the influence of the irrelevant dimension on different responses. For example, for subjects instructed to make judgments solely on the basis of the parts of the object, different responses to stimuli with different parts could be more difficult when the same relation holds across the stimuli (as in the same-relation condition), relative to when the relation also differs across the stimuli (as in the no-same condition). However, the results from a number of studies examining the influence of irrelevant stimuli on different responses have been inconsistent (see Farell, 1985; Garner, 1988), with many studies showing no such effects or diminished effects (e.g., Besner \& Coltheart, 1975; Kimchi, 1988; Miller \& Bauer, 1981; Santee \& Egeth, 1980). Thus, the influence of encoding and maintaining relational information across a saccade on the eye movement trials and within a fixation on the no eye movement trials was expected for same responses, but perhaps not for different responses.

\section{Method}

\section{Subjects}

Sixteen University of Notre Dame undergraduates participated; they were compensated with partial course credit or with monetary payment at a rate of $\$ 6 / \mathrm{h}$. None of the subjects had taken part in Experiment 1 . Eight subjects were randomly assigned to one of two instruction conditions.

\section{Stimuli and Apparatus}

The stimuli and apparatus were the same as those in Experiment 1.

\section{Design}

For each of the 16 figures, there were 12 trials, 3 in the all-same condition, 3 in the same-relation condition, 3 in the same-part condition, and 3 in the no-same condition. This yielded 192 trials. All 192 trials were presented in each of the four overlap conditions (eye movement trials: retinal overlap and spatial overlap; no eye movement trials: retinal + spatial overlap and no overlap). The eye movement trials were randomly divided into two blocks of 96 trials each, with the subjects performing one block in each condition during an experimental session, with order counterbalanced. In an additional session, the subjects performed 192 trials of each type of no eye movement trial. As in Experiment 1, the no eye movement session was conducted after the eye movement session, because the mean saccade latencies were needed for the presentation of the first figure in the no eye movement trials.

There was also a between-subjects instruction manipulation that defined how the subjects responded to the stimulus pairs. The subjects in the relation-instruction group were told to respond same if the relation among the parts remained constant across the two stimuli, regardless of whether the parts remained constant. Thus, these subjects responded same to the all-same and same-relation conditions and different to the same-part and no-same conditions (see the Relation-Instruction column in Figure 1). The subjects in the partinstruction group were told to respond same if the parts remained constant across the two stimuli, regardless of whether the relations among the parts remained constant (see the Part-Instruction column in Figure 1). Thus, these subjects responded same to the all-same and same-part conditions and different to the same-relation and allsame conditions.

\section{Procedure}

Each subject took part in an initial practice session and three experimental sessions. Each of the first two experimental sessions consisted of two blocks of 96 eye movement trials, one block with retinal overlap trials and the other with spatial overlap trials, with the order counterbalanced across days. The criteria for acceptable eye movement trials were the same as those in Experiment 1. For the partinstruction group, $60 \%$ of the trials in the retinal overlap condition (percent eliminated for criteria $1-5: 0 \%, 0 \%, 7 \%, 33 \%$, and $0 \%$, respectively) and $83 \%$ of the trials in the spatial overlap condition (percent eliminated for criteria 1-5: $0 \%, 0 \%, 4 \%, 13 \%$, and $0 \%$, respectively) were acceptable $[t(7)=4.1]$. For the relation-instruction group, $66 \%$ of the trials in the retinal overlap condition (percent eliminated for criteria $1-5: 1 \%, 0 \%, 9 \%, 24 \%$, and $0 \%$, respectively) and $80 \%$ of the trials in the spatial overlap condition (percent eliminated for criteria $1-5: 1 \%, 0 \%, 5 \%, 14 \%$, and $0 \%$, respectively) were acceptable $[t(7)=2.9]$. For both groups, the difficulty in the retinal overlap condition was in moving the eyes off of the foveally presented stimulus to the saccade target; this resulted in small saccades that preceded the saccade to the target (criterion 4). 
The last experimental session consisted of four blocks of 96 trials, two for each type of trial, with the order of the blocks counterbalanced. As in Experiment 1, each subject's mean saccade latency on the retinal overlap eye movement trials $(M \mathrm{~s}=315$ and $357 \mathrm{msec}$ across subjects for the part-instruction group and the relationinstruction group, respectively) determined the duration of the first stimulus for their retinal + spatial overlap no eye movement trials. Similarly, each subject's mean saccade latency on the spatial overlap trials $(M \mathrm{~s}=200$ and $251 \mathrm{msec}$ for the part-instruction and relationinstruction groups, respectively) determined the duration of the first stimulus for the no overlap no eye movement trials. Trials with response times greater than $5,000 \mathrm{msec}$ were considered unacceptable (less than $1 \%$ of the data).

\section{Results and Discussion}

\section{Part-Instruction Group}

Mean correct response times and percent correct for acceptable trials, broken down by response (same or different), pair type, and overlap condition, are presented in Table 2. Separate 4 (overlap condition: retinal overlap eye movement, spatial overlap eye movement, retinal + spatial overlap no eye movement, and no overlap no eye movement) $\times 2$ (pair type) repeated measures ANOVAs were conducted for same and different trials on response times and percent correct.

Same trials. For response times, there was a main effect of pair type, with responses in the all-same condition $(M=448 \mathrm{msec})$ being faster than responses in the partsame condition $\left[M=501 \mathrm{msec} ; F(1,7)=20.5, M S_{\mathrm{e}}=\right.$ $2,196]$. There was also a main effect of overlap condition $\left[F(3,21)=6.5, M S_{\mathrm{e}}=7,320\right]$, with a critical difference of $89 \mathrm{msec}$ being required for significance. Basically, the no eye movement conditions $(M \mathrm{~s}=434$ and $420 \mathrm{msec}$ for the retinal + spatial overlap and no overlap conditions, respectively) were generally faster than the eye movement conditions $(M \mathrm{~s}=523$ and $519 \mathrm{msec}$ for the retinal overlap and spatial overlap conditions, respectively). Impor- tantly, the interaction between overlap condition and pair type was not significant $(F<1)$.

For percent correct, there was a main effect of pair type, with accuracy in the all-same condition $(M=96 \%)$ being significantly higher than accuracy in the part-same condition $\left[M=92 \% ; F(1,7)=7.5, M S_{\mathrm{e}}=27.9\right]$. There was also a marginally significant effect of overlap condition $\left[F(3,21)=2.8, M S_{\mathrm{e}}=14.8, p=.07\right]$. On the basis of a critical difference of $4 \%$ required for significance, accuracy in the retinal overlap eye movement condition $(M=$ $96 \%$ ) was significantly greater than accuracy in the spatial overlap eye movement condition $(M=92 \%)$. The two no eye movement conditions did not differ from each other ( $M=95 \%$ for both the retinal + spatial overlap condition and the no overlap condition) or from either of the eye movement conditions. The interaction between pair type and overlap condition was not significant $(F<1)$.

The pair type effects that were present across all overlap conditions indicate a similar influence of relation information in transsaccadic memory and visual shortterm memory. Despite instructions to selectively attend to the parts, when the relation remained constant across the stimuli (as in the all-same condition), responses were significantly faster and more accurate than when the relation differed across the stimuli (as in the part-same condition).

Different trials. In the response time analysis, there was only a significant effect of overlap condition $[F(3,21)$ $=4.2, M S_{\mathrm{e}}=6,144$; all other $\left.F_{\mathrm{s}}<1.1, p \mathrm{~s}>.38\right]$. On the basis of a critical difference of $82 \mathrm{msec}$ for significance, the only difference was between the no overlap no eye movement condition ( $M=485 \mathrm{msec})$ and the spatial overlap eye movement condition $(M=568 \mathrm{msec})$. The general pattern was faster responses in the no eye movement conditions $(M \mathrm{~s}=485$ and $513 \mathrm{msec}$ for the no overlap and retinal + spatial overlap conditions, respectively) than

Table 2

Mean Correct Response Times (in Milliseconds) and Percent Correct (PC) for Same and Different Responses Broken Down by Trial Type for the Part-Instruction Group in Experiment 2

\begin{tabular}{|c|c|c|c|c|c|c|c|c|}
\hline \multirow[b]{4}{*}{ Trial Type } & \multicolumn{8}{|c|}{ Response and Stimulus Pair Type } \\
\hline & \multicolumn{4}{|c|}{ Same Responses } & \multicolumn{4}{|c|}{ Different Responses } \\
\hline & \multicolumn{2}{|c|}{ All-Same } & \multicolumn{2}{|c|}{ Same-Part } & \multicolumn{2}{|c|}{ Same-Relation } & \multicolumn{2}{|c|}{ No-Same } \\
\hline & $M$ & PC & $M$ & $\mathrm{PC}$ & $M$ & $\mathrm{PC}$ & $M$ & PC \\
\hline \multicolumn{9}{|c|}{ Eye Movement Trials } \\
\hline Retinal overlap & 485 & 97 & 561 & 94 & 574 & 95 & 551 & 98 \\
\hline Spatial overlap & 491 & 95 & 546 & 88 & 564 & 95 & 572 & 94 \\
\hline \multicolumn{9}{|c|}{ No Eye Movement Trials } \\
\hline Retinal + spatial overlap & 414 & 95 & 454 & 94 & 498 & 98 & 529 & 97 \\
\hline No overlap & 399 & 96 & 441 & 93 & 486 & 95 & 484 & 95 \\
\hline
\end{tabular}

Note-All-same refers to a stimulus pair with the same parts and same relation; same-part refers to a stimulus pair with the same parts but different relation; same-relation refers to a stimulus pair with the same relation but different parts; no-same refers to a stimulus pair with different parts and different relations. For this instruction group, participants should respond same to the first two conditions and different to the last two conditions. 
in the eye movement conditions $(M \mathrm{~s}=563$ and $568 \mathrm{msec}$ for the retinal overlap and spatial overlap conditions, respectively).

For percent correct, there was only a marginally significant effect of overlap condition $\left[F(3,21)=3.0, M S_{\mathrm{e}}=\right.$ $9.2, p=.06$; all other $F \mathrm{~s}<1]$. On the basis of a critical difference of $3 \%$ required for significance, none of the pairwise comparisons was significant. However, the general pattern was higher accuracy for the retinal overlap eye movement condition and the retinal + spatial overlap no eye movement condition ( $M \mathrm{~s}=96 \%$ and $97 \%$, respectively) than for the spatial overlap eye movement condition and the no overlap no eye movement condition $(M \mathrm{~s}=$ $95 \%$ and $95 \%$, respectively). This could be due to the fact that the first stimulus was presented peripherally in an uncertain location (either to the left or to the right) in the latter two conditions but foveally in a fixed (central) location in the former two conditions.

\section{Relation-Instruction Group}

Mean correct response times and percent correct for acceptable trials, broken down by response (same or different), pair type, and overlap condition, are presented in Table 3. Separate 4 (overlap condition: retinal overlap eye movement, spatial overlap eye movement, retinal + spatial overlap no eye movement, and no overlap no eye movement) $\times 2$ (pair type) repeated measures ANOVAs were conducted for same and different trials on response times and percent correct.

Same trials. For response times, there was a main effect of pair type, with responses in the all-same condition $(M=476 \mathrm{msec})$ being faster than responses in the partsame condition $\left[M=539 \mathrm{msec} ; F(1,7)=48.7, M S_{\mathrm{e}}=\right.$ 1,295; all other $F \mathrm{~s}<1.4, p \mathrm{~s}>.28]$. For percent correct, there was a main effect of pair type, with accuracy in the all-same condition ( $M=98 \%$ ) being significantly higher than accuracy in the part-same condition $[M=96 \%$;
$F(1,7)=9.9, M S_{\mathrm{e}}=5.8 ;$ all other $\left.F \mathrm{~s}<1.3, p \mathrm{~s}>.28\right]$. These pair type effects indicate an influence of part information. Despite instructions to selectively attend to the relations, when the part remained constant across the stimuli (as in the all-same condition), responses were significantly faster and more accurate than when the parts differed across the stimuli (as in the relation-same condition). Moreover, this influence was constant across eye movement and no eye movement trials.

Different trials. In the response time analysis, there were no significant effects (all $F \mathrm{~s}<1.5, p \mathrm{~s}>.26$ ). For percent correct, there was significant interaction between overlap condition and pair type $\left[F(3,21)=3.8, M S_{\mathrm{e}}=4.7\right.$; all other $F \mathrm{~s}<3.2$, $p \mathrm{~s}>.11$ ]; however, the differences among overlap conditions did not depend on the presence or the absence of an eye movement. Specifically, on the basis of a critical difference of $2 \%$ for significance, the part-same condition was more accurate than the no-same condition for both the spatial overlap eye movement condition ( $M \mathrm{~s}=96 \%$ and $93 \%$, respectively) and the no overlap eye movement condition $(M \mathrm{~s}=98 \%$ and $96 \%$, respectively). However, for the retinal + spatial overlap condition, the no-same condition $(M=98 \%)$ was more accurate than the part-same condition $(M=96 \%)$, and these conditions did not differ for the retinal overlap eye movement condition ( $M \mathrm{~s}=96 \%$ and $97 \%$, respectively).

\section{Summary}

Analyses from both groups support the idea that the encoding and retention of relation and part information is obligatory. The data from the eye movement trials support the idea that the first stimulus is encoded in the form of a structural description, so that it is decomposed into its parts and their constituent relations (Carlson-Radvansky \& Irwin, 1995). This structural description is then accessed upon presentation of the second stimulus for comparison. Importantly, there were no differences between

Table 3

Mean Correct Response Times (in Milliseconds) and Percent Correct (PC) for Same and Different Responses Broken Down by Trial Type for the Relation-Instruction Group in Experiment 2

\begin{tabular}{|c|c|c|c|c|c|c|c|c|}
\hline \multirow[b]{4}{*}{ Trial Type } & \multicolumn{8}{|c|}{ Response and Stimulus Pair Type } \\
\hline & \multicolumn{4}{|c|}{ Same Responses } & \multicolumn{4}{|c|}{ Different Responses } \\
\hline & \multicolumn{2}{|c|}{ All-Same } & \multicolumn{2}{|c|}{ Same-Relation } & \multicolumn{2}{|c|}{ Same-Part } & \multicolumn{2}{|c|}{ No-Same } \\
\hline & $M$ & $\mathrm{PC}$ & $M$ & $\mathrm{PC}$ & $M$ & $\overline{\mathrm{PC}}$ & $M$ & $\mathrm{PC}$ \\
\hline \multicolumn{9}{|c|}{ Eye Movement Trials } \\
\hline Retinal overlap & 484 & 98 & 542 & 94 & 567 & 97 & 580 & 96 \\
\hline Spatial overlap & 495 & 98 & 557 & 95 & 577 & 96 & 590 & 93 \\
\hline \multicolumn{9}{|c|}{ No Eye Movement Trials } \\
\hline Retinal + spatial overlap & 462 & 97 & 531 & 97 & 578 & 96 & 572 & 98 \\
\hline No overlap & 464 & 98 & 526 & 97 & 593 & 98 & 602 & 96 \\
\hline
\end{tabular}

Note-All-same refers to a stimulus pair with the same parts and same relation; same-relation refers to a stimulus pair with the same relation but different parts; same-part refers to a stimulus pair with the same parts but different relation; no-same refers to a stimulus pair with different parts and different relations. For this instruction group, participants should respond same to the first two conditions and different to the last two conditions. 
the eye movement and the no eye movement conditions in terms of the retention of parts and relations, suggesting similar operations within transsaccadic memory and visual short-term memory.

\section{EXPERIMENT 3}

Given the difficulties in separating the processing of parts from the processing of their relations (see, e.g., Kimchi, 1992), in Experiment 3, a different set of stimuli was used to examine the retention of relational information in transsaccadic memory. Specifically, a same/different comparison task was used to compare successive presentations of dot matrices from Irwin (1991). In Irwin's experiments, subjects were shown a dot pattern in one fixation, executed a saccade, and were shown a second dot pattern in the next fixation. The task was to judge whether the patterns were the same or different. The patterns were created by randomly filling in eight positions in a $4 \times 4$ array. On same trials, identical patterns were presented; on different patterns, one dot was randomly selected and moved to a randomly selected unfilled location. Across six experiments, Irwin found that subjects could perform this task with significantly better than chance accuracy with simple and complex patterns, with 6-dot and 10-dot patterns, and in the presence of a spatial displacement of the second pattern, relative to the first. On the basis of these results, he concluded that transsaccadic memory is long-lasting, limited in capacity, and not tied to specific spatial location.

The goal of Experiment 3 was to examine the ability to compare dot matrices across transsaccadic memory when only relational information could be used as the basis of the response. Because dot matrices are randomly constructed patterns, identity emerges only as a function of the relations among the dots. Therefore, the ability of participants in Irwin's (1991) experiments to perform a same/ different comparison task at above chance levels can be taken as evidence of the retention of relational information. Moreover, in two experiments, Irwin used a spatial displacement manipulation in which the dots in the second matrix were offset by one column, relative to the dots in the first matrix, thus changing the absolute positions. He found no consistent different in accuracy between the displaced conditions and conditions in which the matrices remained in the same location.

Experiment 3 employed a stronger test of this idea by adding a manipulation in which the size of the matrices to be compared either remained constant or varied (first matrix small and second large, or first large and second small). The size manipulation changed the absolute location of all of the dots and, thus, rendered comparison of the matrices strictly dependent on relational information. More specifically, this manipulation maintained the qualitative aspect of the relational information, in the sense that one dot remained in the same categorical relation (see, e.g., Kosslyn et al., 1989) to another dot across any size change. However, the coordinate (see, e.g., Koss- lyn et al., 1989) or quantitative relational information was changed, in the sense that dots that were separated by $0.2^{\circ}$ of visual angle in the small matrix were separated by $0.36^{\circ}$ of visual angle in the large matrix. If the nature of the relational information that is preserved includes both qualitative and quantitative aspects, the following two predictions can be made. On the basis of the qualitative aspect, subjects should be able to make sameldifferent judgments of these matrices despite the size change at significantly above chance levels. On the basis of the quantitative aspect, the speed and accuracy with which such judgments are made may vary with the size change. Following the logic of Experiment 2, for same responses, significantly faster and more accurate performance should be observed for same-size matrices than for different-size matrices, because the size dimension is congruent with the same response in the former case but incongruent in the latter case. As in Experiment 2, no systematic pattern was expected for different responses.

Given that even randomly constructed dot matrices may embody some structure such that parts may be constructed out of subsets of the dots, an additional manipulation of matrix complexity was used. Specifically, some of the matrices formed a simple structure that generally allowed an easy segmentation into parts, and some of the matrices were complex, with no discernible structure; samples are shown in Figure 4. The complex matrices thus require the strictest reliance on relational information.

Finally, given the failure to find systematic overlap effects in Experiments 1 and 2, two additional overlap conditions were included, to further test the consistent use of structural descriptions within and across fixations: a peripheral retinal eye movement condition and a peripheral retinal + spatial no eye movement condition. The peripheral retinal eye movement condition involved presenting the first stimulus to the left or the right periphery. Upon

\section{Simple matrices}

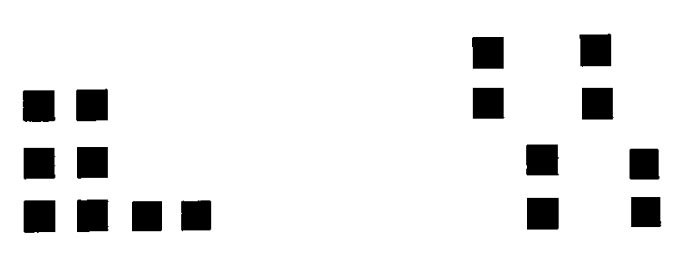

Complex matrices

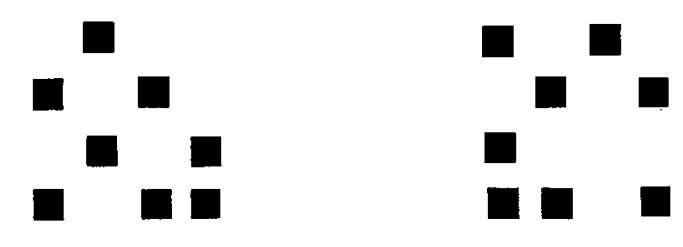

Figure 4. Sample simple and complex matrices used in Experiment 3. 
A

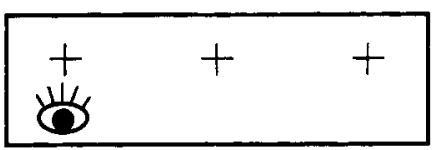

Calibration: Each point fixated for 1.5 seconds

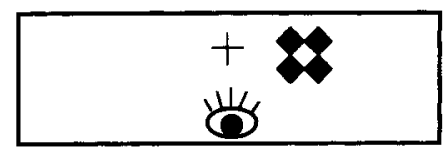

First figure appears in periphery with central fixation target

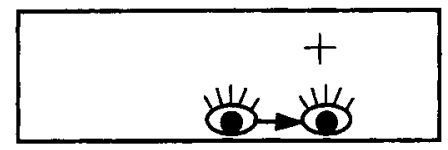

Upon saccade detection first figure disappears for $42 \mathrm{~ms}$

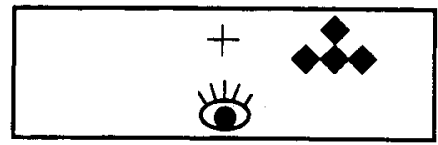

Second figure appears in periphery; response collected
B

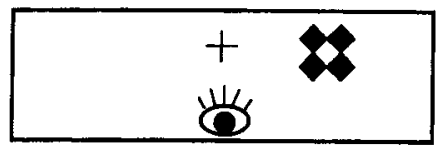

First figure appears in periphery for duration of mean saccade latency from spatial overlap trials

Screen is blank for $42 \mathrm{~ms}$

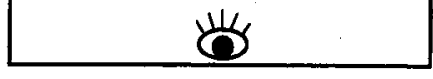
(1)

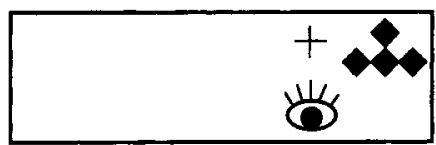

Second figure appears

in periphery;

response collected

Figure 5. Additional eye movement and no eye movement trial types used in Experiment 3. Panel $A$ illustrates the peripheral retinal overlap eye movement trials; panel $B$ illustrates the peripheral no eye movement trials.

elicitation of an eye movement, the second stimulus was presented to the left or the right of the targeted landing site of the eye movement, thus maintaining the location of the two stimuli on the peripheral retina. The peripheral retinal + spatial no eye movement condition involved presenting the first stimulus to the left or the right periphery, with the second stimulus presented in that same location after a $42-\mathrm{msec}$ interval. The two new conditions are illustrated in Figure 5.

\section{Method}

\section{Subjects}

Eight subjects from the University of Notre Dame community were tested; they were compensated at a rate of $\$ 6$ for each hour of their participation. None had participated in Experiments 1 and 2. Data from 1 subject were excluded owing to exceedingly high error rates across all eye movement conditions.

\section{Stimuli and Apparatus}

The apparatus from Experiment 1 was used. Following Irwin (1991, Experiment 6), the stimuli consisted of 64 dot matrices, defined and rated for complexity by Ichikawa (1985). Thirty-two formed a simple structure (patterns 26 through 57 in Table 2 of Ichikawa, 1985) and had a mean complexity rating of 2.83 (out of 7 ; the higher the score, the more complex the pattern), with a range of 2.4-3.3. Thirty-two formed a complex structure (patterns 109 through 140 in Table 2 of Ichikawa, 1985) and had a mean complexity rating of 6.28 (out of 7), with a range 5.9-6.7 (Ichikawa, 1985; see Figure 4 for sample matrices). Small matrices subtended $2.19^{\circ}$ of visual angle horizontally and vertically, and large matrices subtended $2.68^{\circ}$; thus, the size manipulation involved an $18 \%$ change. Dot size was $0.4^{\circ}$ in both the large and the small matrices. Distance from the center of the central matrix to the center of the left and right matrices was $4.75^{\circ}$ of visual angle; this was constant across the small and large matrices, thus requiring the subjects to perform the same size eye movement on all trials. 
64.7], with significantly more trials acceptable in the spatial overlap condition $(M=84 \%$; percent eliminated for criteria $1-5: 2 \%, 0 \%$, $7 \%, 7 \%$, and $0 \%$, respectively) than in both the peripheral retinal overlap condition $[M=73 \%$; percent eliminated for criteria 1-5: $4 \%, 0 \%, 13 \%, 10 \%$, and $0 \%$, respectively; $t(6)=8.2]$, and the foveal retinal overlap condition $[M=65 \%$; percent eliminated for criteria $1-5: 12 \%, 0 \%, 12 \%, 11 \%$, and $0 \%$, respectively; $t(6)=3.8]$. The latter two conditions did not significantly differ $[t(6)=1.6, p=$ .16]. The poorer performance in the foveal retinal condition was due mostly to the difficulty that the subjects had with moving off of the foveally presented figure, resulting in small saccades that preceded the saccade to the target. The poorer performance in the peripheral retinal condition was due mostly to the difficulty that the subjects had with landing accurately on the saccade target.

Table 4

Mean Correct Response Times (in Milliseconds) and Percent Correct (PC) for Experiment 3, Broken Down by Overlap Condition, Response Type, Trial Type, Stimulus Complexity, and Same or Different Size Stimulus Pairs

Relative Size of Stimulus Pairs

Same Different

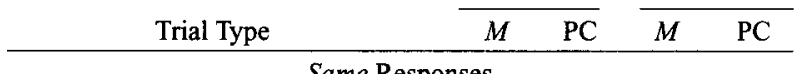

$\begin{array}{lllll}\begin{array}{l}\text { Eye movement conditions } \\ \text { Foveal retinal overlap }\end{array} & & & & \\ \quad \text { Simple matrices } & 497 & 98 & 519 & 97 \\ \quad \text { Complex matrices } & 515 & 92 & 564 & 89 \\ \quad \text { Spatial overlap } & & & & \\ \quad \text { Simple matrices } & 499 & 91 & 531 & 95 \\ \quad \begin{array}{l}\text { Complex matrices } \\ \text { Peripheral retinal overlap }\end{array} & 536 & 84 & 579 & 78 \\ \quad \text { Simple matrices } & 539 & 94 & 561 & 87 \\ \quad \text { Complex matrices } & 551 & 94 & 599 & 79 \\ \text { No eye movement conditions } & & & & \\ \text { Foveal retinal + spatial overlap } & & & & \\ \quad \text { Simple matrices } & 429 & 99 & 495 & 98 \\ \quad \text { Complex matrices } & 464 & 98 & 518 & 97 \\ \text { No overlap } & & & & \\ \quad \text { Simple matrices } & 502 & 99 & 533 & 96 \\ \quad \text { Complex matrices } & 558 & 90 & 609 & 83 \\ \text { Peripheral retinal + spatial overlap } & & & & \\ \quad \text { Simple matrices } & 483 & 98 & 560 & 95 \\ \quad \text { Complex matrices } & 538 & 96 & 677 & 75\end{array}$

Eye movement conditions

Different Responses

\begin{tabular}{lllll} 
Foveal retinal overlap & & & & \\
$\quad$ Simple matrices & 593 & 86 & 581 & 79 \\
$\quad$ Complex matrices & 603 & 78 & 595 & 73 \\
Spatial overlap & & & & \\
$\quad$ Simple matrices & 567 & 84 & 577 & 82 \\
$\quad$ Complex matrices & 575 & 74 & 579 & 75 \\
Peripheral retinal overlap & & & & \\
$\quad$ Simple matrices & 653 & 66 & 655 & 71 \\
$\quad$ Complex matrices & 666 & 55 & 673 & 66 \\
No eye movement conditions & & & & \\
Foveal retinal + spatial overlap & & & & \\
$\quad$ Simple matrices & 548 & 96 & 577 & 95 \\
$\quad$ Complex matrices & 550 & 96 & 617 & 83 \\
No overlap & & & & \\
$\quad$ Simple matrices & 646 & 82 & 618 & 87 \\
$\quad$ Complex matrices & 622 & 68 & 635 & 74 \\
Peripheral retinal + spatial overlap & & & & \\
$\quad$ Simple matrices & 607 & 87 & 605 & 91 \\
$\quad$ Complex matrices & 621 & 84 & 603 & 83 \\
\hline
\end{tabular}

For no eye movement trials, the mean saccade latency for each subject from the appropriate eye movement trials was used to set the duration of the first stimulus. The foveal retinal + spatial overlap no eye movement trials used the mean saccade latency from the foveal retinal overlap eye movement trials $(M=355 \mathrm{msec}$ across all subjects); the no overlap no eye movement trials used the mean saccade latency from the spatial overlap eye movement trials ( $M=264 \mathrm{msec}$ ); and the peripheral retinal + spatial overlap trials used the mean saccade latency from the peripheral retinal overlap trials ( $M=292 \mathrm{msec}$ ).

\section{Results and Discussion}

The results of interest are the mean correct response times and percent correct calculated as a function of response, overlap condition, matrix complexity, and size. These are presented in Table 4. Data for same and different responses were submitted to separate 6 (overlap condition: foveal retinal overlap eye movement, spatial overlap eye movement, peripheral retinal overlap eye movement, foveal retinal + spatial overlap no eye movement, no overlap no eye movement, and peripheral retinal + spatial no eye movement) $\times 2$ (matrix complexity) $\times 2$ (same or different size) repeated measures ANOVAs conducted on responses times and percent correct.

\section{Same Responses}

Response times. There was a main effect of overlap condition $\left[F(5,30)=3.4, M S_{\mathrm{e}}=8,969.8\right]$, a main effect of complexity $\left[F(1,6)=15.3, M S_{\mathrm{e}}=6,026.2\right]$, and a main effect of relative size $\left[F(1,6)=44.5, M S_{\mathrm{e}}=2,633.7\right]$. These were all qualified by three significant two-way interactions; the three-way interaction was not significant $(F<1.3, p>.32)$. First, there was an interaction between overlap condition and complexity $\left[F(5,30)=5.1, M S_{\mathrm{e}}=\right.$ 803.9]. On the basis of a critical difference of $31 \mathrm{msec}$ for significance, five of the overlap conditions exhibited faster responses to the simple figures than to the complex figures (foveal retinal overlap: $M=32-\mathrm{msec}$ difference [complex - simple]; spatial overlap: $M=43-\mathrm{msec}$ difference; foveal retinal + spatial overlap: $M=31-\mathrm{msec}$ difference; no overlap: $M=66-\mathrm{msec}$ difference; and peripheral retinal + spatial overlap: $M=87 \mathrm{msec}$ ), with the conditions with a peripheral presentation of the first figure showing the largest effects; this difference was not significant for the peripheral retinal overlap eye movement ( $M=25$-msec difference). This general pattern of faster responses to simple stimuli than to complex stimuli is consistent with the idea that parts may have been identified within the simple matrices and that this may have facilitated comparison of the stimuli.

Second, there was an interaction between overlap condition and size $\left[F(5,30)=8.1, M S_{\mathrm{e}}=720\right]$. On the basis of a critical difference of $29 \mathrm{msec}$ for significance, performance was more difficult when the matrices were different sizes than when the matrices were the same sizes. Such difficulty was present across all overlap conditions, indicating retention of metric relational information. However, there were larger size effects for the no eye movement 
conditions than for the eye movement conditions (foveal retinal overlap: $M=36$-msec difference [different size same size]; spatial overlap: $M=38$-msec difference; peripheral retinal overlap: $M=35$-msec difference; foveal retinal + spatial overlap: $M=59-\mathrm{msec}$ difference; no overlap: $M=41-\mathrm{msec}$ difference; and peripheral retinal + spatial overlap: $M=109$-msec difference). This is the only difference obtained between eye movement and no eye movement conditions with respect to representation and indicates more exact encoding of quantitative information in visual short-term memory than in transsaccadic memory.

Third, there was a significant interaction between complexity and size $\left[F(1,6)=15.6, M S_{\mathrm{e}}=330.4\right]$. On the basis of a critical difference of $24 \mathrm{msec}$, there was a significant effect of stimulus complexity for same-size stimuli $(M=36-\mathrm{msec}$ difference [complex - simple] and for different-size stimuli ( $M=58$-msec difference). The fact that this effect was much larger for different-size stimuli makes sense, given that complex matrices force a greater reliance on relational information and that the size manipulation disrupts the metric aspect of the relational information.

Percent correct. There was a main effect of overlap condition $\left[F(5,30)=3.3, M S_{\mathrm{e}}=131.6\right]$, a main effect of complexity $\left[F(1,6)=6.2, M S_{\mathrm{e}}=416.1\right]$, and a main effect of relative size $\left[F(1,6)=23.2, M S_{\mathrm{e}}=48.2\right]$. These were all qualified by two significant two-way interactions; all other interactions were not significant $(F<1.9, p>.13)$. First, there was an interaction between overlap condition and size $\left[F(5,30)=3.1, M S_{\mathrm{e}}=55.2\right]$; a difference of $8 \%$ was required for significance. The general pattern was more accurate performance when the matrices were the same size than when they were different sizes, although this effect was significant only in the two peripheral overlap conditions $(M=11 \%$ difference [same size - different size] for both the peripheral retinal overlap eye movement and peripheral retinal + spatial overlap no eye movement conditions; foveal overlap: $M=2 \%$ difference; spatial overlap: $M=2 \%$ difference; foveal retinal + spatial overlap: $M=0 \%$ difference; no overlap: $M=4 \%$ difference). This pattern is consistent with the response time analyses, indicating the maintenance of metric relational information in transsaccadic and visual short-term memory.

Second, there was a significant interaction between complexity and size $\left[F(1,6)=16.6, M S_{\mathrm{e}}=32.1\right]$. On the basis of a critical difference of $7 \%$, there was a significant effect of stimulus complexity for different-size stimuli $(M=12 \%$ difference [simple - complex] $)$, but not for same-size stimuli ( $M=4 \%$ difference). This effect is consistent with the response time analyses, and indicates that a change in metric information was particularly disruptive for complex matrices. Importantly, performance in all the conditions was highly accurate, with even the most difficult condition (complex matrices of different sizes, $M=83 \%$ ) being significantly above chance $[t(6)=$
18.7]. This indicates the successful retention of the qualitative aspects of relational information.

\section{Different Responses}

Response times. There was a main effect of overlap condition $\left[F(5,30)=8.7, M S_{\mathrm{e}}=3,853\right]$ that was qualified by a significant interaction between overlap condition and relative size $\left[F(5,30)=3.7, M S_{\mathrm{e}}=928.9\right]$; a critical difference of $33 \mathrm{msec}$ was required for significance. The effect of relative size was significant for the foveal retinal + spatial overlap condition $(M=48-\mathrm{msec}$ difference [different size - same size]). However, the differences in the other conditions were not significant and exhibited no systematic pattern (foveal retinal overlap: $M=-10$-msec difference; spatial overlap: $M=6$ msec difference; peripheral retinal overlap: $M=4-\mathrm{msec}$ difference; no overlap: $M=-7$-msec difference; peripheral retinal + spatial overlap: $M=-10$-msec difference). There was also a marginally significant main effect of matrix complexity, with response to simple matrices $(M=$ $602 \mathrm{msec}$ ) being faster than responses to complex matrices $\left[M=612 \mathrm{msec} ; F(1,6)=5.3, M S_{\mathrm{e}}=686, p=.06\right]$. There were no other significant effects (all $F \mathrm{~S}<3, p \mathrm{~s}>.13$ ).

Percent correct. There was a main effect of overlap condition $\left[F(5,30)=10.4, M S_{\mathrm{e}}=235.4\right]$ that was qualified by a significant interaction between overlap condition and size $\left[F(5,30)=5.1, M S_{\mathrm{e}}=49.4\right]$; a critical difference of $8 \%$ was required. Accuracy was higher for different-size matrices than for same-size matrices in the peripheral retinal + spatial overlap condition $(M=-8 \%$ difference [different size - same size]). However, the differences in the other conditions were not significant and exhibited no systematic pattern (foveal retinal overlap: $M=6 \%$ difference; spatial overlap: $M=-6 \%$ difference; foveal retinal + spatial overlap: $M=-7 \%$ difference; no overlap: $M=6 \%$ difference; peripheral retinal + spatial overlap: $M=2 \%$ difference). There was also a significant main effect of matrix complexity, with response to simple matrices $(M=84 \%)$ being more accurate than responses to complex matrices $[M=76 \% ; F(1,6)=15.9$, $\left.M S_{\mathrm{e}}=169\right]$. There were no other significant effects (all $F \mathrm{~s}<1.4, p \mathrm{~s}>.25)$.

\section{Summary}

Consistent with Experiment 2, the effects of interest were in the same response trials in which support for the maintenance of both qualitative and quantitative aspects of relational information was obtained. Specifically, the subjects were able to perform the task significantly above chance, indicating a role for the qualitative aspect of relational information. Moreover, their performance on the simple and complex matrices largely mirrored Irwin's (1991) results with the same stimuli. However, the size change manipulation did make the task more difficult, as reflected in longer response times and less accurate performance when the matrices changed size than when they remained the same. This indicates a role for 
the quantitative aspect of relational information. Interestingly, metric information may be better represented in visual short-term memory than in transsaccadic memory, as is suggested by larger size change effects in the response times.

\section{GENERAL DISCUSSION}

On the basis of the retention and successful use of parts across a saccade, Carlson-Radvansky and Irwin (1995) suggested that structural descriptions are a representational form that is used in transsaccadic memory. Because structural descriptions encode not only parts of objects, but also their relations (see, e.g., Gottschaldt, 1926/1967; Palmer, 1977; Sutherland, 1968), the present experiments sought supporting evidence for the transsaccadic use of structural descriptions, by examining whether relational information was also maintained across saccades. In Experiment 1, a same/different comparison task with stimuli that consisted of various parts organized into various configurations was used. A saccade intervened between the presentations of the two stimuli. The data of interest consisted of the three types of different trials: trials in which both the parts and the relations differed (nosame condition), trials in which the parts were the same but the relations differed (same-part condition), and trials in which the relations were the same but the parts differed (same-relation condition). The results showed that responses were generally faster and more accurate in the no-same condition than in either the same-part or the same-relation condition. This indicates that the maintenance of the parts and relations across the stimuli increased the difficulty with which they could be judged as being different and suggests that both types of information were encoded in transsaccadic memory. Moreover, retention of such information did not differ systematically across eye movement or no eye movement trials.

In Experiment 2, the obligatory nature of such encoding was examined by instructing subjects to base their same/different judgments on the basis of one (relevant) dimension while ignoring the other (irrelevant) dimension. The results showed significant influences of the irrelevant dimension on same responses, so that when the irrelevant dimension was inconsistent with the relevant dimensions, there was more difficulty than when the irrelevant dimension was consistent with the relevant dimension. This general pattern held for both part and relation information, both within and across fixations.

Finally, in Experiment 3, a set of dot matrices was used, in which part and relation information were defined in a different manner than in Experiments 1 and 2. Specifically, a size manipulation was adopted that changed the absolute location of all of the dots in the matrix, forcing reliance on relational information. Such a manipulation changed a quantitative (metric) aspect of the relational information in terms of distances between the dots, while preserving the qualitative aspect of the relational information in terms of the spatial relations among the dots. The data of most interest were the same response trials. The finding that accuracy was significantly above chance indicated successful retention of qualitative relational information. The finding that same responses were significantly slower when the matrices changed size than when they stayed the same indicated retention of quantitative relational information. These data thus suggest some initial evidence for the retention of both categorical and coordinate (see, e.g., Kosslyn et al., 1989) relational information in transsaccadic memory.

Finally, across all three experiments, there were no systematic differences that were due to the type of overlap (foveal retinal vs. spatial vs. peripheral retinal) in the eye movement or no eye movement conditions. Consistent with previous work, this suggests that information was not tied to retinal or spatial location (Carlson-Radvansky \& Irwin, 1995; Henderson \& Anes, 1994; Irwin, 1991; Pollatsek, Rayner, \& Henderson, 1990).

\section{The Size Change Effect}

The effect of the size manipulation in Experiment 3 is inconsistent with research by Pollatsek et al. (1984), who failed to find a significant influence of a size change on naming latencies to line drawings of common objects. This discrepancy could be due to the nature of the stimuli. Pollatsek et al. (1984) used Snodgrass and Vanderwart (1980) pictures with high name agreement. In contrast, dot matrices with no previous associations were used in the present work. In addition, in the Pollatsek et al. (1984) study, subjects could rely on the parts of the objects (and their altered relations) to name the objects. In contrast, in the present study, such part information was less available for the simple matrices and not at all available for the complex matrices, rendering performance susceptible to the disruption of relational information caused by the size change. Consistent with this, the effect of the size change was greater for complex matrices than for simple matrices. Thus, it is possible that the effect of a size change depends on the reliance on relational information, relative to part information, to identify the stimuli.

\section{Local Relations Versus Global Configuration}

Across all experiments, the results support the idea that structural descriptions that encode parts and relations are used in transsaccadic memory. As was discussed earlier, there are two ways in which the relational information could be represented within a structural description: It could occur at the level within the hierarchical representation at which the local parts are specified, or it could occur at the top level of the hierarchical representation, at which the global configuration is specified. Considered locally, the relational information can be thought of as intra-object relations of the sort possibly encoded within a viewpoint-invariant representation, such as Marr's (1982) 3-D representation or an objectcentered reference frame. Considered globally, the relational information can be thought of within a global structure that could be specified with respect to a viewer- 
Table 5

Mean Correct Response Times (in Milliseconds) and Percent Correct (PC) for No Eye Movement Trials in Experiment 3, Broken Down by Response Type, Trial Type, Stimulus Complexity, and Same or Different Size Stimulus Pairs

\begin{tabular}{|c|c|c|c|c|}
\hline \multirow[b]{3}{*}{ Trial Type } & \multicolumn{4}{|c|}{ Relative Size of Stimulus Pairs } \\
\hline & \multicolumn{2}{|c|}{ Same } & \multicolumn{2}{|c|}{ Different } \\
\hline & $M$ & PC & $M$ & $\mathrm{PC}$ \\
\hline \multicolumn{5}{|c|}{ Same Responses } \\
\hline \multicolumn{5}{|c|}{ Foveal retinal + spatial overlap } \\
\hline Simple matrices & 429 & 99 & 495 & 98 \\
\hline Complex matrices & 464 & 98 & 518 & 97 \\
\hline \multicolumn{5}{|l|}{ No overlap } \\
\hline Simple matrices & 502 & 99 & 533 & 96 \\
\hline Complex matrices & 558 & 90 & 609 & 83 \\
\hline \multicolumn{5}{|c|}{ Peripheral retinal + spatial overlap } \\
\hline Simple matrices & 483 & 98 & 560 & 95 \\
\hline Complex matrices & 538 & 96 & 677 & 75 \\
\hline \multicolumn{5}{|c|}{ Different Responses } \\
\hline \multicolumn{5}{|c|}{ Foveal retinal + spatial overlap } \\
\hline Simple matrices & 548 & 96 & 577 & 95 \\
\hline Complex matrices & 550 & 96 & 617 & 83 \\
\hline \multicolumn{5}{|l|}{ No overlap } \\
\hline Simple matrices & 646 & 82 & 618 & 87 \\
\hline Complex matrices & 622 & 68 & 635 & 74 \\
\hline \multicolumn{5}{|c|}{ Peripheral retinal + spatial overlap } \\
\hline Simple matrices & 607 & 87 & 605 & 91 \\
\hline Complex matrices & 621 & 84 & 603 & 83 \\
\hline
\end{tabular}

centered reference frame. Indeed, some support for the maintenance of viewpoint-specific information across saccades has been obtained. For example, Henderson and Siefert (1999) used two different tasks to show that left/ right orientation of line drawings of common objects is represented transsaccadically. Similarly, Verfaille, De Troy, and Van Rensbergen (1994) found that in-depth orientation of a point light walker is also represented across saccades. Such orientation information requires a representation that encodes viewpoint-specific relations, such as Marr's $2 \frac{1}{2} 2$-D representation or a viewer-centered reference frame. Thus, the nature of the relational information that is maintained in a structural description seems to be of a rich and varied nature, including categorical and coordinate relations, as well as viewpoint-specific and, perhaps, viewpoint-invariant information.

\section{Transsaccadic Memory and \\ Visual Short-Term Memory}

Across the three experiments, there were no systematic effects of overlap condition, suggesting that the retention of parts and relations is similar both within an eye fixation and across fixations. The only difference between these stores was found in Experiment 3, where the size change effect was larger for the no eye movement conditions than for the eye movement conditions. However, this difference was one of magnitude only, given the significant size effects in the presence of highly accurate performance in all overlap conditions. Thus, as a whole, the data support Irwin's (1991) contention that transsaccadic memory may be the same as visual short-term mem- ory. This initial claim was based on the findings that both memory stores were of limited capacity, long lasting, and impervious to masking. Carlson-Radvansky and Irwin (1995) bolstered this view by showing that the manner in which information was represented was also the same across these stores. It is important to note, however, that this view does not require that the same set of processes operate on this information within and across fixations. Indeed, a recent view of transsaccadic memory assigns a privileged status to the saccade target (McConkie \& Currie, 1996; see, also, Currie, McConkie, CarlsonRadvansky, \& Irwin, in press). Within this view, various features about a to-be-fixated object are encoded that will assist in locating the object during the next fixation. Once the saccade is completed, this locating information is searched for within the region surrounding the landing site, in order to verify that the eyes have landed accurately. Such a search process would not be necessary within a fixation in the absence of saccades. One of the critical questions, then, centers on the nature of the locating information. The evidence indicating that transsaccadic memory and visual short-term memory are the same suggests that such locating information should also be retained within a fixation, although it may not be used (e.g., for search) in quite the same manner. Therefore, research on the retention of information within a fixation should be used to guide work on identifying the components of the locating information.

\section{CONCLUSION}

Consistent evidence for the retention of part and relational information across a saccade was obtained, supporting the idea that structural descriptions are a representational form that can be used in transsaccadic memory (Carlson-Radvansky \& Irwin, 1995). Moreover, such use is similar both within a fixation and across fixations, supporting the idea that transsaccadic memory is the same as visual short-term memory (Irwin, 1991).

\section{REFERENCES}

Besner, D., \& Coltheart, M. (1975). Same-different judgments with words and nonwords: The differential effects of relative size. Memory \& Cognition, 3, 673-677.

CARLSON-RADVANSKY, L. A., \& IRWIN, D. E. (1995). Memory for structural information across eye movements. Journal of Experimental Psychology: Learning, Memory, \& Cognition, 21, 1441-1458.

Currie, C. B., McConkie, G. W., Carlson-Radvansky, L. A., \& IRWIN, D. E. (in press). The role of the saccade target object in the perception of a visually stable world. Perception \& Psychophysics.

FARELL, B. (1985). "Same"-"different" judgments: A review of current controversies in perceptual comparison. Psychological Bulletin, 98 , 419-456.

FISHER, R. A. (1966). The design of experiments (8th ed.). Edinburgh: Oliver \& Boyd.

GARNER, W. R. (1974). The processing of information and structure. Hillsdale, NJ: Erlbaum.

GARNER, W. R. (1988). Facilitation and interference with a separable redundant dimension in stimulus comparison. Perception \& Psychophysics, 44, 321-330.

GotTsCHALDT, K. (1967). Über den Einfluss der Erfarhung die Wahr- 
nehmung von Figuren [On the influence of experience on the perception of figures]. In W. D. Ellis (Ed.), A source book of Gestalt psychology (pp. 261-318). New York: Humanities Press. (Original work published 1926)

HAWKINS, H. L., \& SHIGLEY, R. H. (1972). Irrelevant information and processing mode in speeded discrimination. Journal of Experimental Psychology, 96, 389-395.

Henderson, J. M. (1997). Transsaccadic memory and integration during real-world object identification. Psychological Science, 8, 51-55.

HENDERSON, J. M., \& ANES, M. D. (1994). Roles of object-file review and type priming in visual identification within and across eye fixations. Journal of Experimental Psychology: Human Perception \& Performance, 20, 826-839.

Henderson, J. M., \& Siefert, A. (1999). The influence of enantiomorphic transformation on transsaccadic object integration. Journal of Experimental Psychology: Human Perception \& Performance, 25 , 243-255.

ICHIKAWA, S. (1985). Quantitative and structural factors in the judgment of pattern complexity. Perception \& Psychophysics, 38, 101-109.

IRwIN, D. E. (1991). Information integration across saccadic eye movements. Cognitive Psychology, 23, 420-456.

IRWIN, D. E. (1992a). Perceiving an integrated visual world. In D. E. Meyer \& S. Kornblum (Eds.), Attention and Performance XIV: Synergies in experimental psychology, artificial intelligence, and cognitive neuroscience (pp. 121-142). Cambridge, MA: MIT Press.

IRWIN, D. E. (1992b). Visual memory within and across fixations. In $\mathrm{K}$. Rayner (Ed.), Eye movements and visual cognition: Scene perception and reading (pp. 146-165). New York: Springer-Verlag.

IRWIN, D. E. (1994). On the measurement of phosphor persistence in oscilloscopic displays. Vision Research, 34, 1623.

IrwIN, D. E., YANTIS, S., \& JoNIDES, J. (1983). Evidence against visual integration across saccadic eye movements. Perception \& Psychophysics, 34, 49-57.

KIMCHI, R. (1988). Selective attention to global and local levels in the comparison of hierarchical patterns. Perception \& Psychophysics, 43, 189-198.

KIмCHI, R. (1992). Primacy of wholistic processing and global/local paradigm: A critical review. Psychological Bulletin, 112, 24-38.

Kosslyn, S. M., Koenig, O., Barrett, A., Cave, C. B., Tang, J., \& GABRIELI, J. D. E. (1989). Evidence for two types of spatial representations: Hemispheric specialization for categorical and coordinate relations. Journal of Experimental Psychology: Human Perception \& Performance, 15, 723-735.

LAMB, M. R., \& RoBERTSON, L. C. (1988). The processing of hierarchical stimuli: Effects of retinal locus, locational uncertainty, and stimulus identity. Perception \& Psychophysics, 44, 172-181.

LofTUs, G. R., \& MAsson, M. E. J. (1994). Using confidence intervals in within-subject designs. Psychonomic Bulletin \& Review, 1, 476-490.

MARR, D. (1982). Vision: A computational investigation into the human representation and processing of visual information. San Francisco: Freeman.

Matin, E. (1974). Saccadic suppression: A review and analysis. Psychological Bulletin, 81, 899-917.

MCConkIE, G. W., \& CURRIE, C. B. (1996). Visual stability across saccades while viewing complex pictures. Journal of Experimental Psychology: Human Perception \& Performance, 22, 563-581.

McConkIE, G. W., \& RaYNer, K. (1975). The span of the effective stimulus during a fixation in reading. Perception \& Psychophysics, 17, 578-586.

Miller, J., \& BaUer, D. W. (1981). Irrelevant differences in the "same""different" task. Journal of Experimental Psychology: Human Perception \& Performance, 7, 196-207.

NAvon, D. (1977). Forest before trees: The precedence of global features in visual perception. Cognitive Psychology, 9, 353-383.

NiCKERSON, R. S. (1972). Binary-classification reaction time: A review of some studies of human information-processing capabilities. Psychonomic Monograph Supplements, 4(17, Whole No. 65), 275-317.
O'Regan, J. K., \& Levy-Schoen, A. (1983). Integrating visual information from successive fixations: Does trans-saccadic fusion exist? Vision Research, 23, 765-768.

PALMer, S. E. (1977). Hierarchical structure in perceptual representation. Cognitive Psychology, 9, 441-474.

Phillips, W. A. (1974). On the distinction between sensory storage and short-term visual memory. Perception \& Psychophysics, 16, 283-290.

PollatSEK, A., \& RAYNER, K. (1992). What is integrated across fixations? In K. Rayner (Ed.), Eye movements and visual cognition: Scene perception and reading (pp. 166-191). New York: Springer-Verlag.

Pollatsek, A., Rayner, K., \& Collins, W. E. (1984). Integrating pictorial information across eye movements. Journal of Experimental Psychology: General, 113, 426-442.

Pollatsek, A., Rayner, K., \& Henderson, J. M. (1990). Role of spatial location in the integration of pictorial information across saccades. Journal of Experimental Psychology: Human Perception \& Performance, 16, 199-210.

PomerantZ, J. R. (1983). Global and local precedence: Selective attention in form and motion perception. Journal of Experimental Psychology: General, 112, 511-535.

RAYNER, K., \& POLLATSEK, A. (1983). Is visual information integrated across saccades? Perception \& Psychophysics, 34, 39-48.

SANTEE, J. L., \& EgETH, H. E. (1980). Selective attention in the speeded classification and comparison of multidimensional stimuli. Perception \& Psychophysics, 28, 191-204.

SnOdGrass, J. C., \& VANDERWART, M. (1980). A standardized set of 260 pictures: Norms for name agreement, image agreement, familiarity, and visual complexity. Journal of Experimental Psychology: Human Learning \& Memory, 6, 174-215.

SUTHERLAND, N. S. (1968). Outlines of a theory of visual pattern recognition in animals and man. Proceedings of the Royal Society: Series $B, 171,297-317$.

Verfaillie, K., De Troy, A., \& Van Rensbergen, J. (1994). Transaccadic integration of biological motion. Journal of Experimental Psychology: Learning, Memory, \& Cognition, 20, 649-670.

\section{NOTES}

1. Specifically, 7 subjects performed the following task. On 50 trials, a sample figure taken from Experiments 1 and 2 was drawn onto a video page and displayed behind a closed shutter. On 50 different trials, no such figure was displayed. For all the trials, the computer then switched to a blank video page, thereby erasing the stimulus (if present). After a 14-msec pause, the shutter opened, and the subject's task was to indicate whether the figure was displayed. The subjects were told that a figure was displayed on half of the trials and that they should distribute their responses so that they indicated that it was present on roughly half of the trials. Hit and false alarm rates were calculated to determine $d^{\prime}$. The mean $d^{\prime}$ was -.34 , and the $95 \%$ confidence interval associated with this mean spanned zero $(-.76$ to +.08$)$, indicating that sensitivity did not differ as a function of whether the figure was presented behind the shutter. Thus, given the timing criteria specified in the Procedure section, phosphor persistence did not contribute to performance.

2 . Some eye movements may have occurred on the no eye movement trials; however, because the eyes were not tracked, such trials could not be identified and eliminated. Using a methodology with similar saccade distance and stimulus characteristics, Carlson-Radvansky and Irwin (1995) eliminated $22 \%$ of the no eye movement trials, owing to a failure to maintain a single fixation on the first stimulus. This is an overestimate of the actual number of saccades that occurred, because it includes trials eliminated on account of blinks. Accordingly, it is reasonable to assume that saccades did not occur on a large majority of no eye movement trials, thus yielding a reasonable index of the retention of relational information in visual short-term memory.

(Manuscript received January 22, 1998; revision accepted for publication June 22, 1998.) 